\title{
1 A universal microfluidic approach for quantitative study of
}

\section{bacterial biofilms}

3 Yuzhen Zhang ${ }^{1,2}$, Lingbin Zeng ${ }^{1,7}$, Yumin Cai $^{1,7}$, Zhaoyuan Chen $^{1,2,6}$, Peng Liu ${ }^{3}$, Luyan Z. Ma ${ }^{4,5}$,

4

$5 \quad{ }^{1}$ Center for Infectious Disease Research, School of Medicine, Tsinghua University, Beijing

6

7

8

9

10

$$
\text { Jintao } \operatorname{Liu}^{1,2^{*}}
$$

100084, China

$$
{ }^{2} \text { Tsinghua-Peking Center for Life Sciences, Beijing 100084, China }
$$

${ }^{3}$ Department of Biomedical Engineering, School of Medicine, Tsinghua University, Beijing 100084, China

${ }^{4}$ State Key Laboratory of Microbial Resources, Institute of Microbiology, Chinese Academy of Sciences, Beijing, 100101, China.

${ }^{5}$ University of Chinese Academy of Sciences, Beijing, 100049, China ${ }^{6}$ Present address: Ningbo Institute of Life and Health Industry, University of Chinese Academy of Sciences, Ningbo 315020, China

${ }^{7}$ These authors contributed equally

*Correspondence: JintaoLiu@tsinghua.edu.cn 
Abstract: Bacteria usually live in densely packed communities called biofilms, where interactions between the bacteria give rise to complex properties. Quantitative analysis is indispensable in understanding those properties. However, current biofilm culturing approaches impose various limitations to these types of analysis. Here, we developed a microfluidic approach for quantitative study of biofilms, which is universal and can be used to culture biofilms of various bacterial species. To demonstrate the advantages of this approach, we present two examples, both of which revealed new biological insights. In the first example, we explored the response of Escherichia coli biofilms to exogenous hydrogen peroxide; We found the biofilms gained resistance to $\mathrm{H}_{2} \mathrm{O}_{2}$, but their growth was slowed down due to the metabolic cost of maintaining the resistance; However, under oxygen limitation, $\mathrm{H}_{2} \mathrm{O}_{2}$ can anti-intuitively boost biofilm growth. In the second example, we explored resource retention by Pseudomonas aeruginosa biofilms; We observed a fluorescent substance within the biofilm and identified it as the siderophore pyoverdine; We further showed that the extracellular matrix component Psl acted as a retention barrier for pyoverdine, minimizing its loss into the environment and therefore potentially promoting sharing of pyoverdine within the biofilm.

Introduction

Bacteria usually live in communities, which is beneficial for their survival ${ }^{1-3}$. Many communities exist on surfaces, where the bacteria secrete extracellular polymeric substances such as proteins, polysaccharides, and extracellular DNA, which enable the bacteria to form dense aggregates ${ }^{4}$. These aggregates are called biofilms. It was estimated that biofilms accounted for the majority of the bacteria in nature ${ }^{5}$. In addition to being environmentally 
cause of infections. Since biofilms are highly tolerant to antimicrobials and to our immune system, those infections are often persistent and are hard to cure ${ }^{6}$.

A distinguishing feature of biofilms is spatial heterogeneity ${ }^{7}$. Due to the dense packing of the bacteria, spatial gradients emerge within biofilms. For example, biofilm periphery is relatively nutrient rich while biofilm interior is nutrient poor; On the other hand, metabolic by-products accumulate at biofilm interior and dissipate toward biofilm periphery. These spatial gradients dictate that bacteria at different locations of the biofilm experience different environments. As a consequence, the bacteria display a broad spectrum of physiological states within a single biofilm, and different regions of the biofilm can have distinct properties ${ }^{8-13}$. Moreover, recent studies found that interactions between different regions of a biofilm gave rise to emergent collective behaviors that are beneficial to the survival of the bacteria ${ }^{14-16}$. Therefore, systematic studies on the spatial heterogeneity can help us to gain a deeper understanding of biofilms and their resistance.

We developed a microfluidic approach of culturing biofilms, which facilitates quantitative analysis. In our previous effort, we adapted a commercial microfluidic chip that was designed for yeast single-cell studies to the culturing of Bacillus subtilis biofilms ${ }^{14}$. However, it is unsuitable for many other species of bacteria, especially those of medical importance. The main reason is that many bacteria display strong preference for adhesion, which causes unintended seeding of bacteria at random locations and leads to clogging of the microfluidic chip, hindering long-term tracking of biofilm properties. Here we developed a new microfluidic design from scratch, which overcame the seeding issue and can be applied to all the commonly studied bacterial species. Moreover, the microfluidic chip can be 
manufactured in a standard microbiology laboratory, and can be easily modified to accommodate the needs of specific experiments. In the following, we will describe our new design, and will also give two examples to demonstrate how our method could be used to reveal new biological insights.

\section{Results}

Description and characterization of the method. Figure 1a is a schematic diagram of our microfluidic chip. A key component is a specially designed bacteria seeding zone 7 on the side of the biofilm growth chamber 3 (Fig. 1a, marked in red; Methods). During loading, we inject planktonic bacteria culture into the loading port 5; The injection pressure creates a narrow gap at the seeding zone, allowing bacteria to pass through; Some of the bacteria are trapped at the seeding zone, while the rest are flushed out through the waste outlet 4 (Fig. 1b, Methods). In this way, we could plant bacteria specifically at the seeding zone. The trapped bacteria later proliferate into the growth chamber, where fresh medium is continuously supplied. By secreting extracellular matrix, the bacteria are able to form a stable and densely packed biofilm in the presence of flow (Fig. 1c). Figure 1d shows time-lapse images of an $E$. coli biofilm, which emerged out of the seeding zone $\sim 8$ hours after loading, and later grew into a round shaped biofilm containing millions of bacteria (Supplementary Video 1).

We used a thin growth chamber ( $6 \mu \mathrm{m}$ thick). In comparison, the radius of the biofilm is on the order of $10^{2}-10^{3} \mu \mathrm{m}$. Therefore, the biofilm is a pancake-like colony with uniform

87 thickness (Fig. 1d). A major advantage of this design is that the spatial and dynamical properties of the biofilm could be easily quantified. We analyzed the growth of the $E$. coli biofilm, and found that its radius increased at a constant rate (defined as biofilm growth rate) 
(Fig. 1e). Image analysis revealed that growth was mainly from the periphery region of the biofilm (Fig. 1f, Methods), and the depth of the growth zone was constant over time (Fig. 1g). The biofilm was cultured with M63B1, a defined minimal medium using glucose as the carbon source ${ }^{17}$. Since nutrients penetrate the biofilm through diffusion (Fig. 1d), and are consumed by the densely packed bacteria during the process, there is an inward gradient of nutrients in the biofilm. Therefore, the growth arrest at biofilm interior should be due to nutrient limitation. We found the depth of the growth zone increased with glucose concentration (Supplementary Fig. 1a), suggesting glucose was the limiting nutrient. Interestingly, we also observed a proportional relationship between biofilm growth rate and the depth of the growth zone (Fig. 1h). Finally, we confirmed that the biofilm growth rate was independent of the chamber thickness (Supplementary Fig. 1b), which gives flexibility to the design of the microfluidic chip.

Another important aspect is the maintenance of oxygen gradient in the biofilm. There are steep $\mathrm{O}_{2}$ gradients in natural biofilms, and biofilm interior is usually hypoxic. Oxygen gradient plays important roles in biofilm physiology ${ }^{11,18}$. Therefore, we need to preserve it in our microfluidic chip. However, a technical issue is that the polydimethylsiloxane (PDMS) material commonly used in microfluidics is gas permeable, which leads to vertical diffusion of $\mathrm{O}_{2}$ from the ambient environment into the growth chamber (Supplementary Fig. 2a), causing loss of

$\mathrm{O}_{2}$ gradient. To overcome this problem, we sealed the PDMS with cover glass and epoxy resin (Fig. 2a), both of which are gas impermeable; The glass covers the chamber portion of the PDMS, so that the biofilm could be observed with microscope; The epoxy resin covers the rest of the device, which also provides mechanical support to the tubes. In addition, we made the 
of the growth chamber), which minimizes lateral diffusion of $\mathrm{O}_{2}$ from the flowing medium through PDMS into biofilm interior. To test the effectiveness of this solution, we imbedded the oxygen probe PtTFPP into the PDMS. The fluorescence of the probe is quenched by $\mathrm{O}_{2}$,

117 which can be used as a measure of $\mathrm{O}_{2}$ availability ${ }^{19}$. Results confirmed that an $\mathrm{O}_{2}$ gradient was generated by the biofilm (Fig. 2b). To test whether the biofilm interior was hypoxic, we utilized the fluorescent protein YFP, which requires $\mathrm{O}_{2}$ to maturate into its fluorescent form 20; We constructed a YFP expressing $E$. coli strain driven by the $p t s G$ promoter; The promoter is activated by glucose limitation and therefore the YFP would mainly be expressed at biofilm interior ${ }^{21}$. Biofilms formed by this strain only showed background fluorescence in our microfluidic chip (Fig. 2c and d, at $34 \mathrm{~h}$ ). We reasoned that the YFP protein was expressed at biofilm interior but did not maturate due to lack of oxygen. Indeed, biofilms cultured without oxygen limitation displayed the expected fluorescence distribution (Supplementary Fig. 2b and $\mathrm{c}$, at $34 \mathrm{~h}$ ). To confirm the existence of immature YFP protein, we inhibited the synthesis of new YFP using the antibiotic tigecycline (Fig. 2d, Supplementary Fig. $2 \mathrm{c}$ ) and then fed air through the loading channel (Fig. 1a, Methods); YFP fluorescence immediately emerged upon feeding air (Fig. 2c and d). Finally, we confirmed that YFP could mature at the periphery region of the biofilm without feeding air (Supplementary Fig. 3). These results showed that the biofilm periphery had access to oxygen, while the biofilm interior was hypoxic.

Biofilm resistance to oxidative stress. To demonstrate how our method could be used to study biofilms, here we present two examples. The first example is on biofilm resistance. Biofilms are known to show high phenotypic resistance to environmental stresses ${ }^{6,22,23}$. We investigated the response of $E$. coli biofilms to exogenous hydrogen peroxide, which induces oxidative stress in bacteria and causes DNA damage ${ }^{24}$. Biofilm growth rate quickly dropped 
to zero upon switching to medium containing $0.03 \% \mathrm{H}_{2} \mathrm{O}_{2}$ (Fig. 3a). However, the biofilm resumed growth after less than 6 hours, showing acquisition of resistance. We monitored the oxidative stress within the biofilm using the reactive oxygen species (ROS) probe DCFH-DA; It showed that $\mathrm{H}_{2} \mathrm{O}_{2}$ was able to affect the periphery region of the biofilm, but had no significant effect on the interior region (Fig. 3b). In E. coli, the primary defense machinery against $\mathrm{H}_{2} \mathrm{O}_{2}$

143 is catalase, which is an enzyme that degrades $\mathrm{H}_{2} \mathrm{O}_{2}$ into $\mathrm{H}_{2} \mathrm{O}$ and $\mathrm{O}_{2}{ }^{24}$. Therefore, we

144 monitored the promoter activity of the catalase gene katG using the fluorescent protein mRFP.

145 We found that katG was mainly expressed in a narrow region next to the stressed biofilm

146 periphery (Fig. 3b). The narrow size of the catalase band suggested that it was an effective

147 barrier in blocking $\mathrm{H}_{2} \mathrm{O}_{2}$ penetration. Since there was no significant effect on the interior

148 region of the biofilm, we wondered why it did not recover to its pre- $\mathrm{H}_{2} \mathrm{O}_{2}$ growth rate. We speculated that the presence of $\mathrm{H}_{2} \mathrm{O}_{2}$ in the medium imposed a metabolic burden on the biofilm; Specially, although catalase does not consume energy when degrading $\mathrm{H}_{2} \mathrm{O}_{2}$, its lifetime could be limited; Therefore, in order to maintain the $\mathrm{H}_{2} \mathrm{O}_{2}$ barrier, the biofilm had to constantly produce new catalase. Consistent with our speculation, when we removed glucose from the medium, the catalase barrier quickly disappeared and the DCFH-DA band spread toward the biofilm interior (Fig. 3c, Supplementary Fig. 4).

We wondered whether external stress always slowed down biofilm growth. Surprisingly,

157 when we used glycerol instead of glucose as the carbon source, $\mathrm{H}_{2} \mathrm{O}_{2}$ counterintuitively boosted biofilm growth (Fig. 3d). The increase of growth rate suggested that $\mathrm{H}_{2} \mathrm{O}_{2}$ might relieved a bottleneck for growth. We wondered whether the bottleneck was oxygen, as degradation of $\mathrm{H}_{2} \mathrm{O}_{2}$ by the catalase barrier could provide additional oxygen to the biofilm.

161 Consistent with our speculation, when we fed air through the loading channel, biofilm growth 
rate was significantly increased (Fig. 3e); In contrast, doubling the concentration of the carbon

163

source glycerol or the nitrogen source $\mathrm{NH}_{4}{ }^{+}$did not have noticeable effect (Supplementary

Fig. 5); Finally, when we cultured the biofilm without oxygen limitation (Supplementary Fig.

2a), the growth was suppressed by $\mathrm{H}_{2} \mathrm{O}_{2}$ (Fig. 3f). It is conceivable that this anti-intuitive boosting of growth by stress is not limited to $\mathrm{H}_{2} \mathrm{O}_{2}$. For example, in addition to concentration, diffusion is another important factor affecting nutrient availability inside biofilm; Therefore, one is likely to boost growth if the stress increased the diffusion or transport of the limiting nutrient into the biofilm.

Resource retention by biofilm. Our method is universal and can be applied to bacteria of various species. In addition to E. coli, we also cultured biofilms with Salmonella typhimurium, P. aeruginosa, Klebsiella pneumoniae, B. subtilis, Staphylococcus aureus, Enterococcus faecium, and Mycobacterium smegmatis (Supplementary Fig. 6), which cover Gram-negative, Gram-positive and mycobacteria. As the second example, here we show our findings on $P$. aeruginosa biofilms. We observed autofluorescence at the interior region of the biofilm under the CFP channel (Fig. 4a). To identify the source of this fluorescence, we took advantage of a well-known behavior of $P$. aeruginosa biofilms - dispersion ${ }^{25}$. In our experiments, when the biofilm reached the size of $\sim 240 \mu \mathrm{m}$, the interior cells escaped through an opening at biofilm periphery, leaving behind a hollow shell (Fig. 4a, Supplementary Video 2); Accompanying the dispersion was the simultaneous disappearance of the fluorescence, suggesting loss of the fluorescent substance into the environment; The biofilm later resealed the opening at its periphery, and the fluorescence re-emerged; Interestingly, we observed fluorescence in the hollow region of the resealed biofilm (Fig. $4 \mathrm{a}$, at $13 \mathrm{~h} 10 \mathrm{~m}$ ). These results suggested that the fluorescent substance was extracellular and was diffusible. 
Since the fluorescence was most intense at biofilm interior, we suspected it might be related

with resource limitation. Using planktonic culture, we confirmed that the autofluorescence emerged when the bacteria entered stationary phase (Fig. 4b). Centrifugation of the stationary phase culture showed that the fluorescence was from the supernatant (Fig. 4c), which confirmed the abovementioned observation that the fluorescent substance in the known to be fluorescent ${ }^{26}$. Spectroscopic analysis showed that the excitation and emission spectrum of the stationary phase supernatant was consistent with that of the pyoverdine (Supplementary Fig. 7). Finally, when we knocked out the pyoverdine synthesis gene $p v d A$, the autofluorescence in the biofilm and in the planktonic culture was reduced to the background level (Fig. 4d), confirming the identity of the fluorescent substance as pyoverdine.

Pyoverdine is a siderophore, which is secreted by the bacteria to chelate irons from the environment. Since the process requires re-uptake, uncontrolled loss of pyoverdine into the environment would be energetically inefficient. Therefore, we wondered whether the biofilm was able to confine pyoverdine within its boundary and limit the loss to the flowing medium.

A potential mechanism for retention is through the extracellular matrix $4,27,28$. Ideally, the matrix should not limit diffusion/sharing of pyoverdine within the biofilm. One matrix component that satisfies this criteria is the polysaccharide Psl, which is mainly distributed at biofilm periphery ${ }^{12,29}$. Therefore, we wondered whether Psl limited the loss of pyoverdine. First, we stained the biofilm using the fluorescently labeled lectin HHA-FITC, which binds specifically to $\mathrm{Psl}{ }^{29}$. Consistent with previous results, fluorescence imaging showed that Psl was mainly distributed at the periphery region of the biofilm (Fig. 4e, Supplementary Fig. 8a); 
by the planktonic cells released from the biofilm ${ }^{30}$. We then treated the biofilm with cellulase

211 - an enzyme that can hydrolyze Psl ${ }^{29}$, both the HHA-FITC and the pyoverdine fluorescence rapidly dropped to a lower level (Fig. $4 \mathrm{f}$ and g, Supplementary Video 3); Cellulase did not affect of pyoverdine. In contrast, treatment of the biofilm with DNase degraded the extracellular eventually boosted Psl level in the biofilm, which is consistent with previous findings that the bacteria could sense the loss of Psl and increase its synthesis via the master regulator c-diGMP 26 .

\section{Discussion}

223

A commonly accepted method of studying biofilms is the flow cell approach, which utilizes a macro fluidic chamber and the biofilms are attached to the chamber wall ${ }^{31}$. It mimics biofilms in flowing environments, which represents a major fraction of natural conditions ${ }^{5}$. Due to the large size of the chamber, biofilms formed in flow cell are usually three-dimensional. In contrast, in our microfluidic approach, we made the growth chamber only a few micrometers thick, so that the biofilms are semi-two-dimensional. Despite the difference, our approach is compatible with the flow cell approach. In fact, the biofilm in our microfluidic device is equivalent to a slice of the biofilm in the flow cell. This is because essential properties such as extracellular environments and cellular states are determined by the distance from biofilm surface instead of lateral position. Indeed, we were able to reproduce phenomena observed in flow cells, such as the dispersion of $P$. aeruginosa biofilms and the spatial distribution of 
Psl (Fig. 4a and e) ${ }^{29}$. Furthermore, we were able to perform quantitative measurements on spatiotemporal dynamics. Quantitative analysis had been uncommon in the biofilm research community, and a major obstacle was the morphological complexity of the biofilms in the commonly used culturing approaches ${ }^{32,33}$. By simplifying the biofilm, we were able to eliminate this obstacle. It is true that morphological complexity could play a role in natural settings. Here we have captured a key feature of natural biofilms - spatial heterogeneity. Therefore, findings based on our approach should be generalizable to more complex scenarios.

Quantitative studies of bacterial communities have mainly been limited to small population size ${ }^{21,34-37}$. However, some important properties are emergent ones that require a minimum population size ${ }^{14-16,38,39}$. It has been challenging to culture large bacteria communities in microfluidics, as the fluidic chambers and channels are prone to clogging by the bacteria ${ }^{40}$. Here we are able to culture bacterial communities containing over a million cells for extended period of time (2-5 days), and maintain precise control over the growth environment throughout the entire duration. The key to our success is a specially designed mechanism for the controlled seeding of bacteria at the micro scale. By separating the loading channel from

251 the growth chamber and only seeding bacteria to the designated cell trap, we avoided the main cause of clogging - random adhesion of bacteria in the growth chamber. The controlled seeding also leads to higher reproducibility of biofilms between different experiments, which is essential for quantitative analysis. Since the seeding mechanism does not rely on the properties of the bacteria, our approach is universal and can be used to culture all the commonly studied bacterial species and potentially many more. 
Our microfluidic approach is easy to implement. The entire process - from design to soft lithography to chip making - could be carried out in a standard microbiology laboratory (Methods). Yet, it is also very flexible, and could be extended to study more complex bacterial communities. For example, one could use it to study interactions between biofilms of different species (Supplementary Fig. 10). Finally, in addition to combining it with microscopic observation, we have realized its potential to achieve more, and we are currently taking advantage of modern molecular biology tools to conduct systems levels investigations on bacterial communities.

Acknowledgements: We thank Jing-ren Zhang and Babak Javid for kindly providing bacterial strains and technical supports, thank Jing-ren Zhang for helpful comments on the manuscript, thank members of the Liu Lab for numerous discussions. JL was supported by the Tsinghua University Independent Research Program (20197030008) and the Tsinghua-Peking Center for Life Sciences, YZ was supported by the National Nature Science Foundation of China (21908129), Chinese Postdoctoral Science Foundation (2018M631481), and the TsinghuaPeking Center for Life Sciences.

273

Author Contributions: $\mathrm{YZ}$ and JL designed the study, $\mathrm{YZ}$ and JL designed the microfluidic chip, $Y Z, L Z, Y C$ and $Z C$ performed the experiments, $Y Z, L Z, Y C$ and JL analyzed the data, $Y Z$ and JL wrote the manuscript, all authors discussed the manuscript.

Competing Interests: $\mathrm{YZ}$ and $\mathrm{JL}$ are inventors of a patent application related with this work.

Data availability: The data that support the findings of this study are available from the corresponding author upon reasonable request. 


\section{Methods}

282

283

284

Microfluidic chip fabrication. The microfluidic chip was designed and manufactured in-house.

A scaled drawing of the microfluidic design is shown in Supplementary Fig. 11. The master mold of the chip was fabricated using the Maskless Mold Fabrication System from BlackHole Lab (France), and performing lithography on glass slides using photoresist from Microchemicals (AZ4562). The microfluidic chips were made with polydimethylsiloxane (PDMS) and glass slides. The PDMS part was made by pouring 10:1 (v/v) mixture of Sylgard 184 elastomer and curing agent (Dow Corning, USA) on the master mold, and curing the mixture for 2 hours in an oven at $80^{\circ} \mathrm{C}$. The cured PDMS was carefully peeled off from the mold and punched with holes at the inlet and outlet ports. Then we pasted a narrow (0.5-1 $\mathrm{mm}$ ) strip of $3 \mathrm{M}$ Scotch Magic tape to a designated location of the PDMS (on the barrier between the growth chamber and the loading channel, and perpendicular to the barrier), and treated the PDMS with plasma for 2 mins (SoftLithoBox, BlackHole Lab, France). After the plasma treatment, the tape was quickly removed, and the PDMS was immediately bounded with a glass slide or cover glass (depending on the magnification of microscopic observations later on). The tape prevented the plasma from reaching the covered spot, therefore, creating an unbounded region between the growth chamber and the loading channel, which could be used for bacteria seeding. Finally, we bounded the top surface of the PDMS (middle portion) with cover glass, plugged the inlet and outlet ports with syringe needles (20G, $0.91 \mathrm{~mm}$ OD x $0.61 \mathrm{~mm} \mathrm{ID)}$ and PTFE Tubing (1/16" OD $\times 1 / 32 \mathrm{ID})$, and sealed the rest of the PDMS with epoxy resin (Fig. 2a).

To visualize the spatial gradient of oxygen (Fig. 2b), we embedded the oxygen sensor PtTFPP (Frontier Scientific Inc., USA) in PDMS: The PtTFPP was dissolved in toluene and thoroughly 
305

306

307

308

309

310

311

312

313

314

315

316

317

318

319

320

321

322

323

324

325

mixed with PDMS before it was cured; Then the mixture was poured on the mold, and toluene was allowed to evaporate while the PDMS was cured. The final PtTFPP concentration was 1 $\mathrm{mg} / \mathrm{ml}$.

Bacterial Strains. The bacterial strains used in this study are Escherichia coli BW25113, Salmonella typhimurium ATCC 14028, Bacillus subtilis NCIB 3610, Pseudomonas aeruginosa PAO1 (LpaaP), Klebsiella pneumoniae ATCC BAA-1144, Staphylococcus aureus RN4220, Enterococcus faecium ATCC 35667, and Mycobacterium smegmatis mc ${ }^{2}-155$ (Supplementary Table 1).

E. coli strains: Promoters of $E$. coli strains (PptsG and PkatG) were amplified from wild-type $E$. coli BW25113 genomic DNA; The PtetR sequence was synthesis by Genewiz (China). The promoter sequences were amplified by PCR and were then introduced to a plasmid containing fluorescent protein coding sequence to generate promoter activity reporters. Plasmids were constructed using the Gibson assembly method and transformed into DH5 $\alpha$ competent cells (weidi, China). DNA purification and isolation of plasmids were performed using reagents from Omega Bio-Tek (USA). PCR reactions were carried out using Q5 High-Fidelity DNA polymerase (NEB, UK).

\section{P. aeruginosa strains: Most of the experiments were based on the PAO1 $\triangle$ paaP stain, which} was less prone to dispersion than wild-type PAO1 and more suitable for the current study; We have verified that paaP knockout does not affect the production of the fluorescent substance (data not shown). The pvdA deletion strain were constructed by an unmarked, nonpolar deletion strategy as previously described (Environ. Microbiol. Rep. 10, 583-593, 2018). 
Bacteria loading and biofilm culturing. On the day before the experiment, bacteria from $-80^{\circ} \mathrm{C}$ glycerol stock were streaked on LB agar plates and incubated at $37{ }^{\circ} \mathrm{C}$ overnight. On the next day, a single colony was picked from the plate and inoculated to $5 \mathrm{ml}$ of LB broth in a $50 \mathrm{ml}$ conical tube, and incubated at $37{ }^{\circ} \mathrm{C}$ in a shaker. After $12 \mathrm{~h}$ of incubation, the culture was centrifuged at a relative centrifugal force of $7000 \mathrm{~g}$ for $3 \mathrm{~min}$, and then the pellet was resuspended in $0.5 \mathrm{ml}$ biofilm medium and used for loading into microfluidics. The biofilm was M63B1 (100 mM KH $2 \mathrm{PO}_{4}, 15 \mathrm{mM}\left(\mathrm{NH}_{4}\right)_{2} \mathrm{SO}_{4}, 0.8 \mathrm{mM} \mathrm{MgSO}_{4}, 3 \mu \mathrm{M}$ vitamin $\mathrm{B} 1,22 \mathrm{mM}$ glucose, adjusted to $\mathrm{pH} 7.4$ with $\mathrm{KOH}){ }^{17}$; The medium for $P$. aeruginosa was LB without sodium chloride (LBNS) ${ }^{29}$; The medium for S. aureus was TSB.

The microfluidic chip was controlled using the Elveflow OB1 Mk3 Pressure Controller (ELVESYS, France), which drives medium flow with pressurized air. At first, the medium was pumped into the growth chamber from ports 1 and 5 (Fig. 1a) simultaneously, with ports 2 and 6 closed. The pressure was maintained at 15 psi until all the air bubbles disappeared, which usually took 5-10 mins. Then port 5 was switched to planktonic bacteria culture with a pressure of 10-20 psi, while port 1 was maintained at 15 psi; Once bacteria started to enter the growth

347 chamber, the pressures to ports 1 and 5 were adjusted to 1-5 psi and 1-2 psi respectively.

348 After $\sim 1000$ bacteria cells were trapped at the seeding zone, we turned off the pressure to port 5, and adjusted the pressure to port 1 to 3 psi. Finally, we opened port 6 and washed the loading channel by injecting PBS to port 5 for 30 mins ( 3 psi). During the rest of the experiment, ports 2, 5 and 6 were kept closed, and port 1 was fed with medium under a constant pressure of 3 psi, and the temperature was kept at $37^{\circ}$ C. To perform perturbations (e.g., adding $\mathrm{H}_{2} \mathrm{O}_{2}$ 
or antibiotic), port 1 was switched to the corresponding new medium, and port 2 was briefly opened until the residues from the previous medium was completely flushed out and the new medium reached port 1 (this operation allowed fast switching of medium). Normally, the biofilm acquires oxygen from the flowing medium; To supply additional oxygen to the interior region of the biofilm (Fig. 2c-d and Fig. 3e), air was pumped into port 5, with port 6 kept open, and the air could then diffuse into the biofilm from the loading channel; To stop the additional oxygen supply, the loading channel was refilled with PBS and ports 5 and 6 were closed.

Time-lapse microscopy. The biofilms were observed with phase contrast and fluorescence microscopy. The microscope used was Olympus IX83 (Japan) with Andor's Zyla 4.2 sCMOS camera (UK). To image the entire biofilm, 10X objective lens was used in most of the experiments. Images were taken every $3 \mathrm{~s}-20 \mathrm{~min}$, depending on the question.

Data analysis. ImageJ (National Institutes of Health, USA) and MATLAB (MathWorks, USA) differencing on snapshots of the biofilm from time-lapse microscopic images; Specifically, we compared the difference between two consecutive phase contrast images by calculating the absolute difference between corresponding pixels in the two images; Since expansion leads to pixel level changes and no expansion leads to little change, image differencing could reveal region of expansion in the biofilm.

374 Planktonic culture experiments. The culturing and measurement of planktonic $P$. aeruginosa 375 (Fig. 4b-e) were performed using the SPARK microplate reader (TECAN, Swiss). The bacteria 376 were grown overnight in the LBNS medium in a $37^{\circ} \mathrm{C}$ shaker. On the next day, $4 \mu \mathrm{l}$ of the 
377 culture were inoculated to $200 \mu \mathrm{l}$ of fresh LBNS and incubated in 96-well plates. Optical

378 density $(600 \mathrm{~nm})$ and fluorescence intensity (excitation $430 \mathrm{~nm}$, emission $480 \mathrm{~nm}$ ) were

379 recorded every $10 \mathrm{~min}$. The excitation and emission spectrum of the supernatant (Fig. 4d)

380 were also measured using the plate reader.

381 


\section{References}

383

1. Lyons, N. A. \& Kolter, R. On the evolution of bacterial multicellularity. Curr. Opin. Microbiol. 24, 21-28 (2015).

2. Meredith, H. R., Srimani, J. K., Lee, A. J., Lopatkin, A. J. \& You, L. Collective antibiotic tolerance: mechanisms, dynamics and intervention. Nat. Chem. Biol. 11, 182-188 (2015).

3. Mukherjee, S. \& Bassler, B. L. Bacterial quorum sensing in complex and dynamically changing environments. Nat. Rev. Microbiol. 17, 371-382 (2019).

4. Flemming, H.-C. \& Wingender, J. The biofilm matrix. Nat. Rev. Microbiol. 8, 623-633 (2010).

5. Flemming, H.-C. \& Wuertz, S. Bacteria and archaea on Earth and their abundance in biofilms. Nat. Rev. Microbiol. 17, 247-260 (2019).

6. Costerton, J. W., Stewart, P. S. \& Greenberg, E. P. Bacterial Biofilms: A Common Cause of Persistent Infections. Science 284, 1318-1322 (1999).

7. Stewart, P. S. \& Franklin, M. J. Physiological heterogeneity in biofilms. Nat. Rev. Microbiol. 6, 199-210 (2008).

8. Klausen, M., Aaes-Jørgensen, A., Molin, S. \& Tolker-Nielsen, T. Involvement of bacterial migration in the development of complex multicellular structures in Pseudomonas aeruginosa biofilms. Mol. Microbiol. 50, 61-68 (2003).

9. Klauck, G., Serra, D. O., Possling, A. \& Hengge, R. Spatial organization of different sigma factor activities and c-di-GMP signalling within the three-dimensional landscape of a bacterial biofilm. Open Biol. 8, 180066 (2018).

10. Srinivasan, S. et al. Matrix Production and Sporulation in Bacillus subtilis Biofilms Localize to Propagating Wave Fronts. Biophys. J. 114, 1490-1498 (2018). 
405

406

407

408

409

410

411

412

413

414

415

416

417

418

419

420

421

422

423

424

425

426

11. Schiessl, K. T. et al. Phenazine production promotes antibiotic tolerance and metabolic heterogeneity in Pseudomonas aeruginosa biofilms. Nat. Commun. 10, 762 (2019).

12. Passos da Silva, D. et al. The Pseudomonas aeruginosa lectin LecB binds to the exopolysaccharide Psl and stabilizes the biofilm matrix. Nat. Commun. 10, 2183 (2019).

13. Jorth, P., Spero, M. A., Livingston, J. \& Newman, D. K. Quantitative Visualization of Gene Expression in Mucoid and Nonmucoid Pseudomonas aeruginosa Aggregates Reveals Localized Peak Expression of Alginate in the Hypoxic Zone. mBio 10, (2019).

14. Liu, J. et al. Metabolic co-dependence gives rise to collective oscillations within biofilms. Nature 523, 550-554 (2015).

15. Prindle, A. et al. Ion channels enable electrical communication in bacterial communities. Nature 527, 59-63 (2015).

16. Liu, J. et al. Coupling between distant biofilms and emergence of nutrient timesharing. Science 356, 638-642 (2017).

17. Chalabaev, S. et al. Biofilms Formed by Gram-Negative Bacteria Undergo Increased Lipid A Palmitoylation, Enhancing In Vivo Survival. mBio 5, (2014).

18. Wessel, A. K. et al. Oxygen Limitation within a Bacterial Aggregate. mBio 5, e0099214 (2014).

19. Ungerböck, B., Charwat, V., Ertl, P. \& Mayr, T. Microfluidic oxygen imaging using integrated optical sensor layers and a color camera. Lab. Chip 13, 1593-1601 (2013).

20. Shaner, N. C., Steinbach, P. A. \& Tsien, R. Y. A guide to choosing fluorescent proteins. Nat. Methods 2, 905-909 (2005). 
21. Dal Co, A., Ackermann, M. \& van Vliet, S. Metabolic activity affects the response of single cells to a nutrient switch in structured populations. J. R. Soc. Interface 16, 20190182 (2019).

22. Hall, C. W. \& Mah, T.-F. Molecular mechanisms of biofilm-based antibiotic resistance and tolerance in pathogenic bacteria. FEMS Microbiol. Rev. 41, 276-301 (2017).

23. Yan, J. \& Bassler, B. L. Surviving as a Community: Antibiotic Tolerance and Persistence in Bacterial Biofilms. Cell Host Microbe 26, 15-21 (2019).

24. Imlay, J. A. The molecular mechanisms and physiological consequences of oxidative stress: lessons from a model bacterium. Nat. Rev. Microbiol. 11, 443-454 (2013).

25. Rumbaugh, K. P. \& Sauer, K. Biofilm dispersion. Nat. Rev. Microbiol. 18, 571-586 (2020).

26. Chen, Y. et al. Multiple diguanylate cyclase-coordinated regulation of pyoverdine synthesis in Pseudomonas aeruginosa. Environ. Microbiol. Rep. 7, 498-507 (2015).

27. Dragoš, A. \& Kovács, Á. T. The Peculiar Functions of the Bacterial Extracellular Matrix. Trends Microbiol. 25, 257-266 (2017).

28. Saunders, S. H. et al. Extracellular DNA Promotes Efficient Extracellular Electron Transfer by Pyocyanin in Pseudomonas aeruginosa Biofilms. Cell 182, 919-932.e19 (2020).

29. Ma, L. et al. Assembly and Development of the Pseudomonas aeruginosa Biofilm Matrix. PLOS Pathog. 5, e1000354 (2009).

30. Zhao, K. et al. Psl trails guide exploration and microcolony formation in Pseudomonas aeruginosa biofilms. Nature 497, 388-391 (2013).

31. Christensen, B. B. et al. Molecular tools for study of biofilm physiology. Methods Enzymol. Acad. Press 310, 20-42 (1999). 
450

451

452

453

454

455

456

457

458

459

460

461

462

463

464

465

466

467

468

469

470

471

32. Asally, M. et al. Localized cell death focuses mechanical forces during 3D patterning in a biofilm. Proc. Natl. Acad. Sci. 109, 18891-18896 (2012).

33. Kragh, K. N., Alhede, M., Kvich, L. \& Bjarnsholt, T. Into the well-A close look at the complex structures of a microtiter biofilm and the crystal violet assay. Biofilm 1, 100006 (2019).

34. Qin, B. et al. Cell position fates and collective fountain flow in bacterial biofilms revealed by light-sheet microscopy. Science 369, 71-77 (2020).

35. Bittihn, P., Didovyk, A., Tsimring, L. S. \& Hasty, J. Genetically engineered control of phenotypic structure in microbial colonies. Nat. Microbiol. 5, 697-705 (2020).

36. Alnahhas, R. N. et al. Majority sensing in synthetic microbial consortia. Nat. Commun. 11, 3659 (2020).

37. Hartmann, R. et al. Quantitative image analysis of microbial communities with BiofilmQ. Nat. Microbiol. 6, 151-156 (2021).

38. Gregor, T., Fujimoto, K., Masaki, N. \& Sawai, S. The Onset of Collective Behavior in Social Amoebae. Science 328, 1021-1025 (2010).

39. Cao, Y. et al. Collective Space-Sensing Coordinates Pattern Scaling in Engineered Bacteria. Cell 165, 620-630 (2016).

40. Drescher, K., Shen, Y., Bassler, B. L. \& Stone, H. A. Biofilm streamers cause catastrophic disruption of flow with consequences for environmental and medical systems. Proc. Natl. Acad. Sci. 110, 4345-4350 (2013). 
a

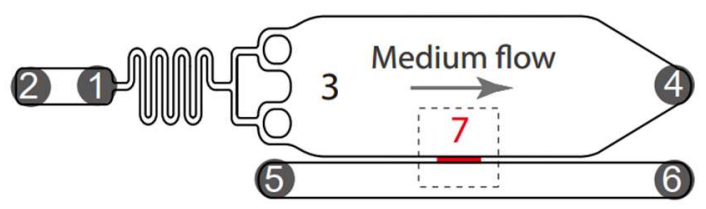

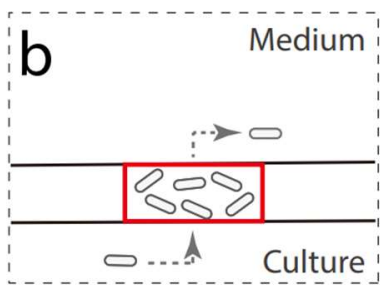

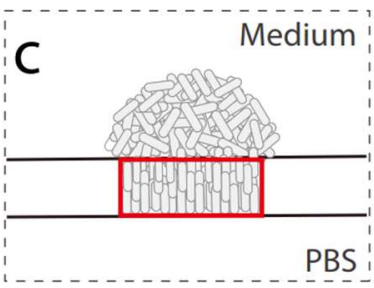

472
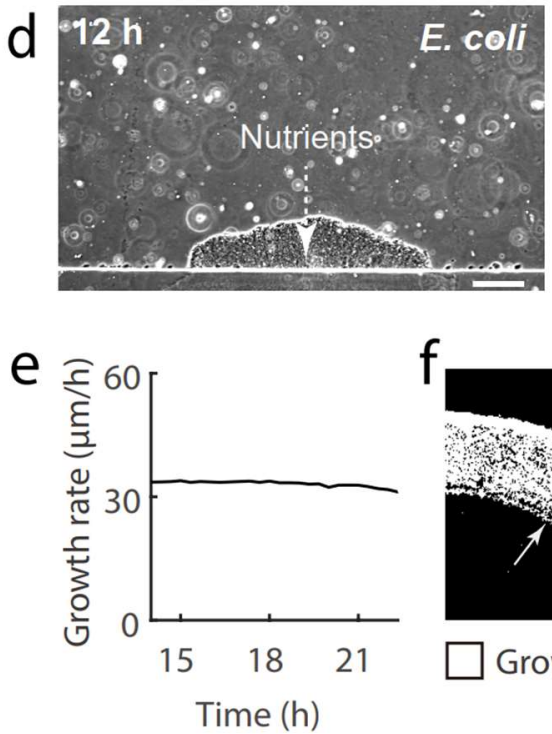

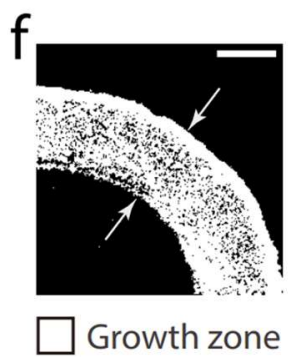

Growth zone
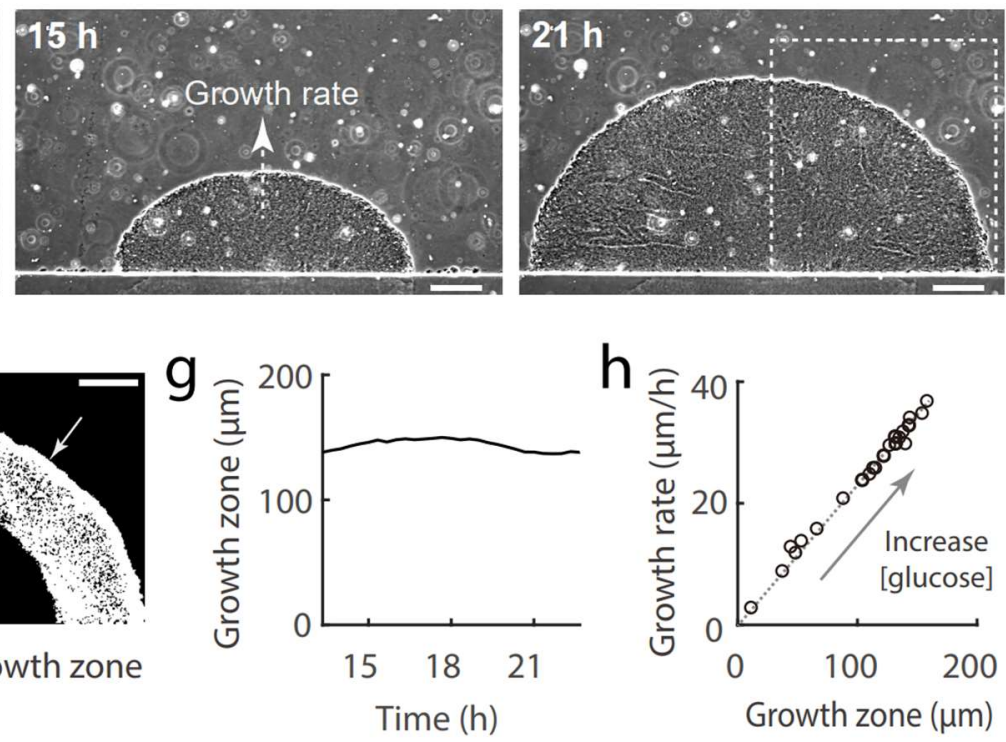

473 Fig. 1: Microfluidic design and growth of E. coli biofilm. a, Schematic diagram of the

474 microfluidic device: 1) medium inlet; 2) medium exchange port; 3) growth chamber; 4) waste outlet; 5 \& 6) bacteria loading channel and ports; 7) bacteria seeding zone (marked in red). $\mathbf{b}$,

Schematic diagram of the bacteria loading process: The dashed rectangular region in $\mathbf{a}$ is shown; Planktonic culture of bacteria were injected into the loading channel; Some of the bacteria were squeezed into the seeding zone (marked in red) by the injection pressure, and those that were squeezed through were flushed out through the waste outlet. c, Schematic diagram of a biofilm growing out of the seeding zone. $\mathbf{d}$, Phase contrast images of a growing E. coli biofilm; Three representative time points are shown; Scale bar, $100 \mu \mathrm{m}$. e, The growth rate of the biofilm shown in $\mathbf{d}$. $\mathbf{f}$, Differencing analysis of the phase contrast images (Methods) revealed zone of growth in biofilm; The dashed rectangular region in $\mathbf{d}$ is shown; Scale bar, $100 \mu \mathrm{m} . \mathbf{g}$, The width of the growth zone shown in $\mathbf{f} . \mathbf{h}$, Biofilm growth rate was proportional to the width of the growth zone, and the two were determined by glucose concentration. 
a

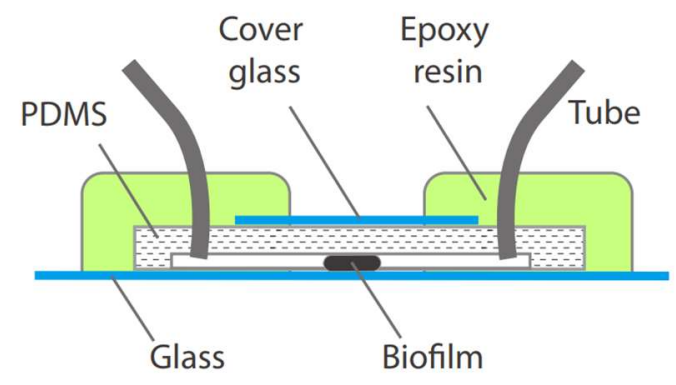

b

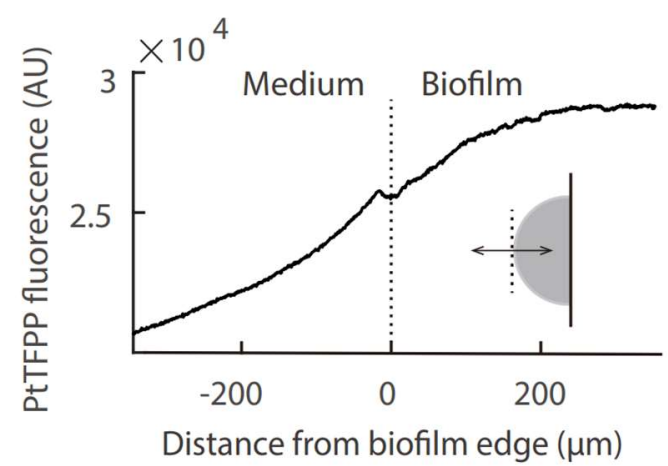

C
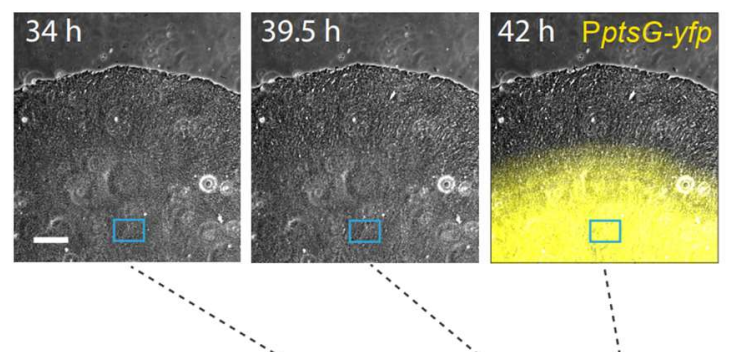

d

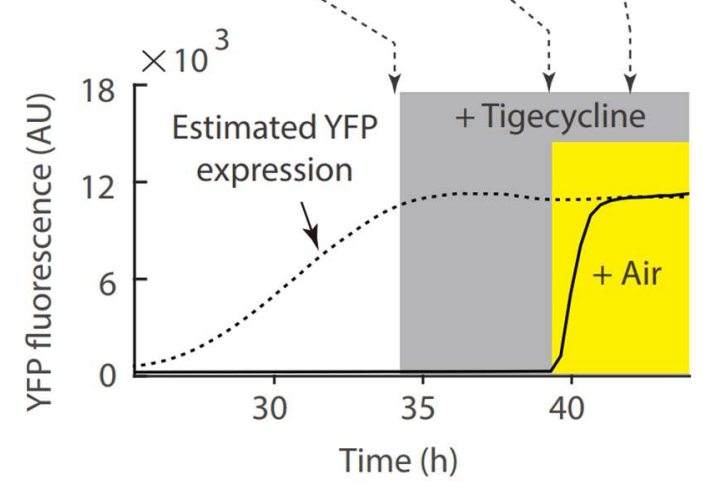

Fig. 2: Maintenance of oxygen gradient. a, Side view of the microfluidic chip. b, Spatial profile of PtTFPP fluorescence over an E. coli biofilm; The double arrow in the inset illustrates the line along which the profile was measured. c, Time-lapse images of an E. coli biofilm; Composite of phase contrast and YFP (shown in yellow) channels; The YFP channel reveals the activity of the ptsG promoter; Scale bar, $100 \mu \mathrm{m}$. d, YFP fluorescence intensity at biofilm interior (the region marked by the rectangle in c; The solid line shows the measured fluorescence value;

493 The dashed line shows the estimated level of YFP protein based on the dashed line in 494 Supplementary Fig. 2c; The shaded regions represent the durations of tigecycline $(2 \mu \mathrm{g} / \mathrm{ml}$, 495 shown in gray) and air (shown in yellow) treatments respectively; The dashed arrows indicate 496 the time when the snapshots in c were taken. 

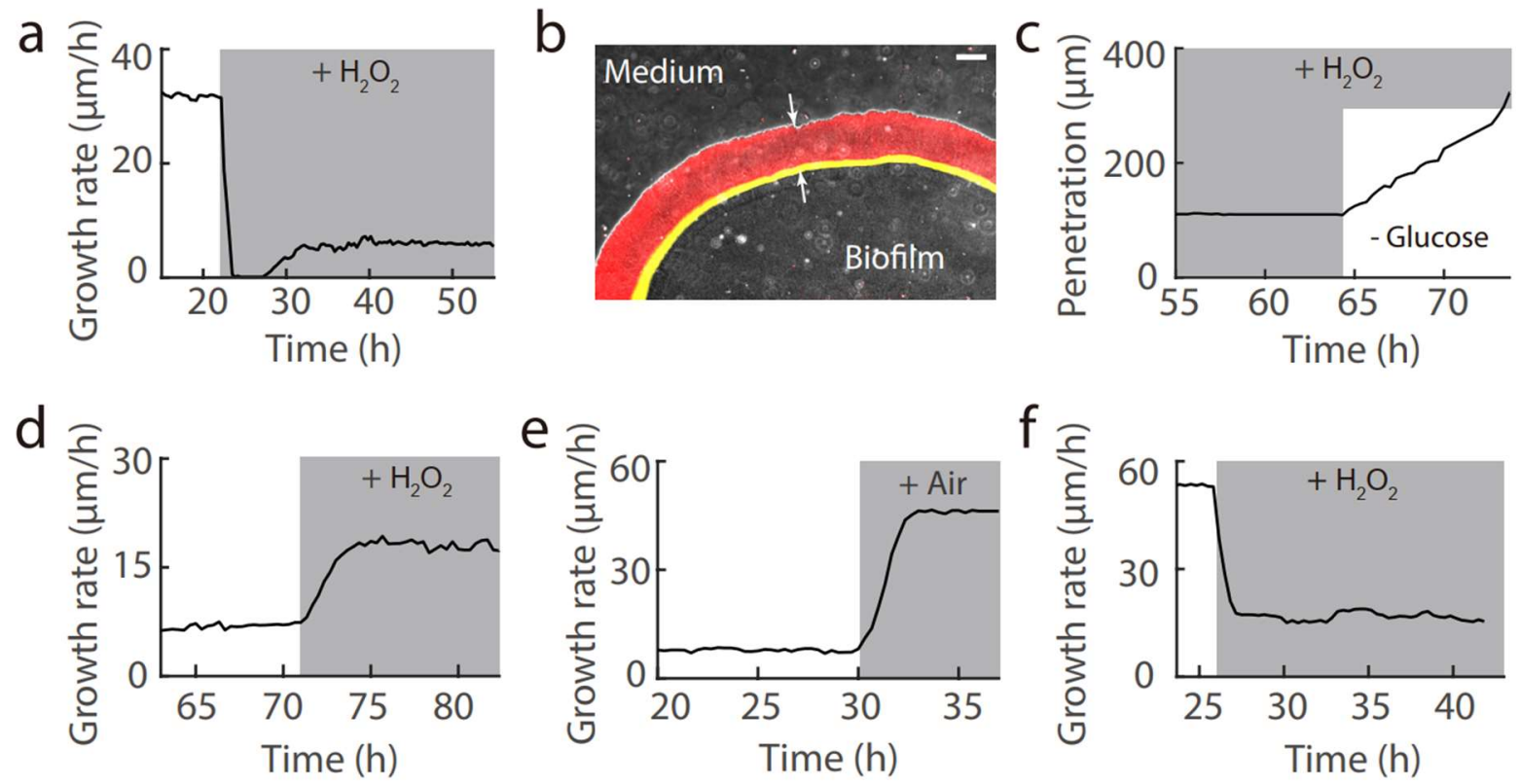

499 Fig. 3: Resistance of E. coli biofilm to exogeneous $\mathbf{H}_{\mathbf{2}} \mathbf{O}_{2}$. a, Biofilm growth rate before and

500 after switching to medium containing $0.03 \% \mathrm{H}_{2} \mathrm{O}_{2}$. b, Snapshot of biofilm after forming

501 resistance to $\mathrm{H}_{2} \mathrm{O}_{2}$; Composite of phase contrast, YFP (DCFH-DA fluorescence, shown in red), and RFP (PkatG-mrfp fluorescence, shown in yellow) channels; The arrows indicate the region

503 of biofilm penetrated by $\mathrm{H}_{2} \mathrm{O}_{2}$; Scale bar, $100 \mu \mathrm{m}$. c, Depth of $\mathrm{H}_{2} \mathrm{O}_{2}$ penetration before and 504 after removing glucose from the medium. d-f, Biofilms cultured with modified M63B1

505 medium - using glycerol (44 mM) instead of glucose as the carbon source. d, Biofilm response

506 to $0.03 \% \mathrm{H}_{2} \mathrm{O}_{2}$. e, Biofilm response to supplementation of air through the loading channel. $\mathbf{f}$,

507 Biofilm response to $0.03 \% \mathrm{H}_{2} \mathrm{O}_{2}$, cultured without oxygen limitation (Supplementary Fig. 2a). 
a

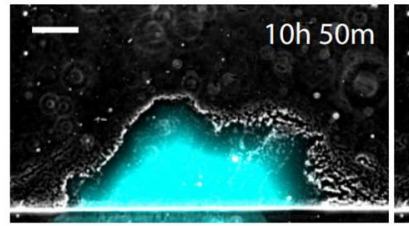

b

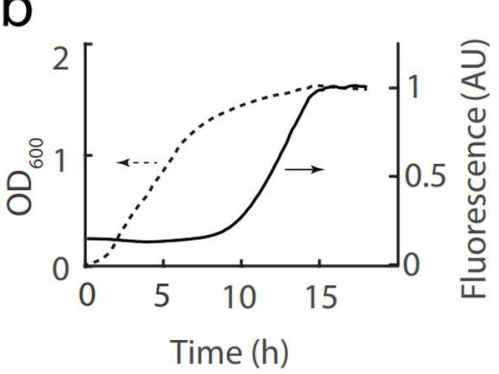

e

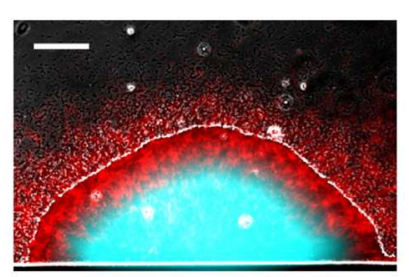

509
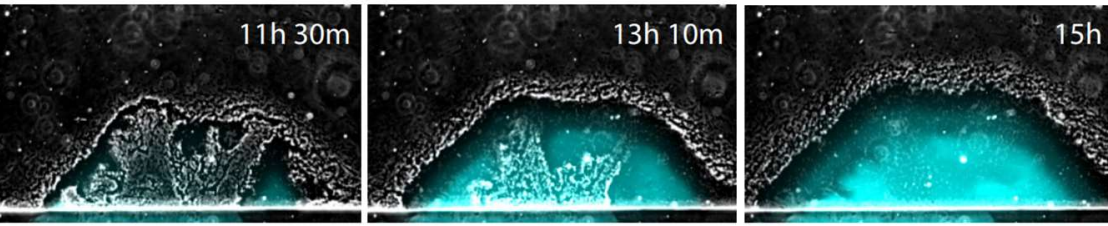

C

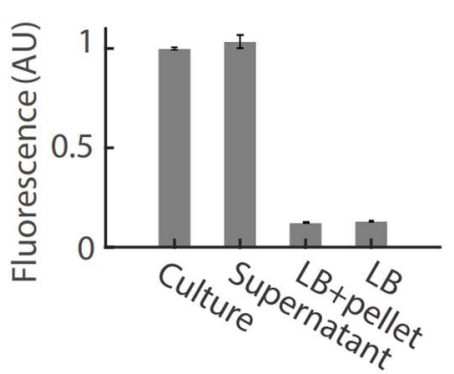

d

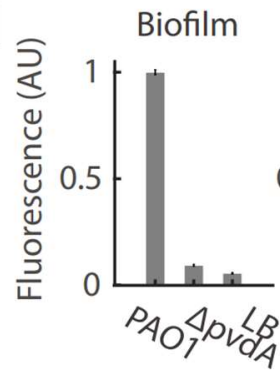

Planktonic

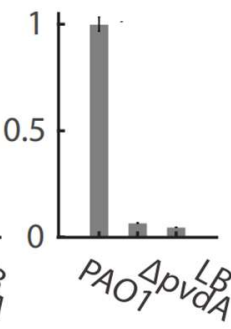

g

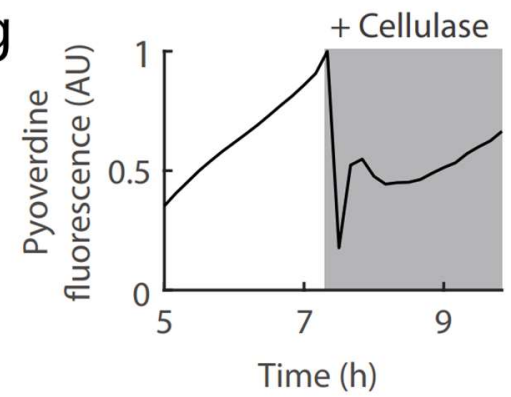

Fig. 4: Resource retention by $P$. aeruginosa biofilm. a, Time-lapse images of a $P$. aeruginosa

biofilm; Composite of phase contrast and CFP (shown in cyan) channels; Scale bar, $100 \mu \mathrm{m} . \mathbf{b}$,

Optical density and fluorescence intensity (CFP channel) of planktonic bacteria culture. c, Fluorescence intensities of stationary phase planktonic bacteria culture, its supernatant after centrifugation, its resuspended pellet after centrifugation, and the resuspension medium LB; Error bars represent standard deviation, $n=3$ biological replicates. $\mathbf{d}$, Fluorescence intensities of biofilm (interior region) and stationary phase planktonic cultures with or without $p v d A$ knockout. e, Staining of biofilm by the fluorescent dye HHA-FITC (shown in red, $100 \mathrm{ug} / \mathrm{ml}$ ), which binds specifically to the extracellular matrix component Psl; Scale bar, $100 \mu \mathrm{m}$. f, HHAFITC fluorescence at biofilm periphery before and after switching to medium containing cellulase (84 U/ml, Sigma C2730-50ML). g. Pyoverdine fluorescence at biofilm interior. 

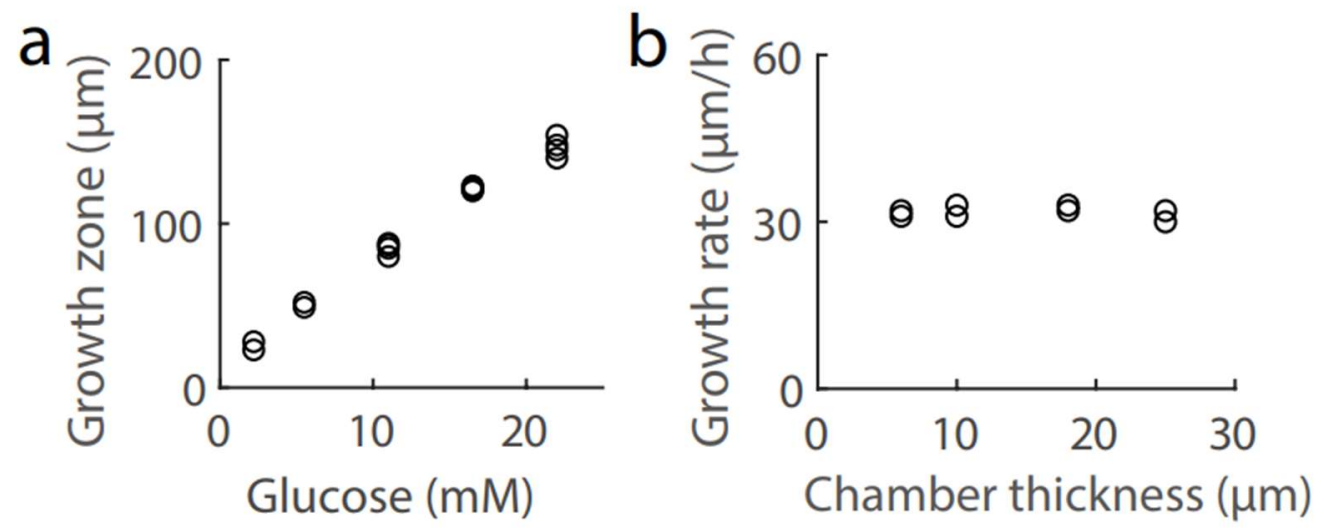

523 Supplementary Fig. 1: Growth of $E$. coli biofilm. a, The depth of the growth zone increased

524 with glucose concentration. b, Biofilm growth rate was independent of chamber thickness.

525 

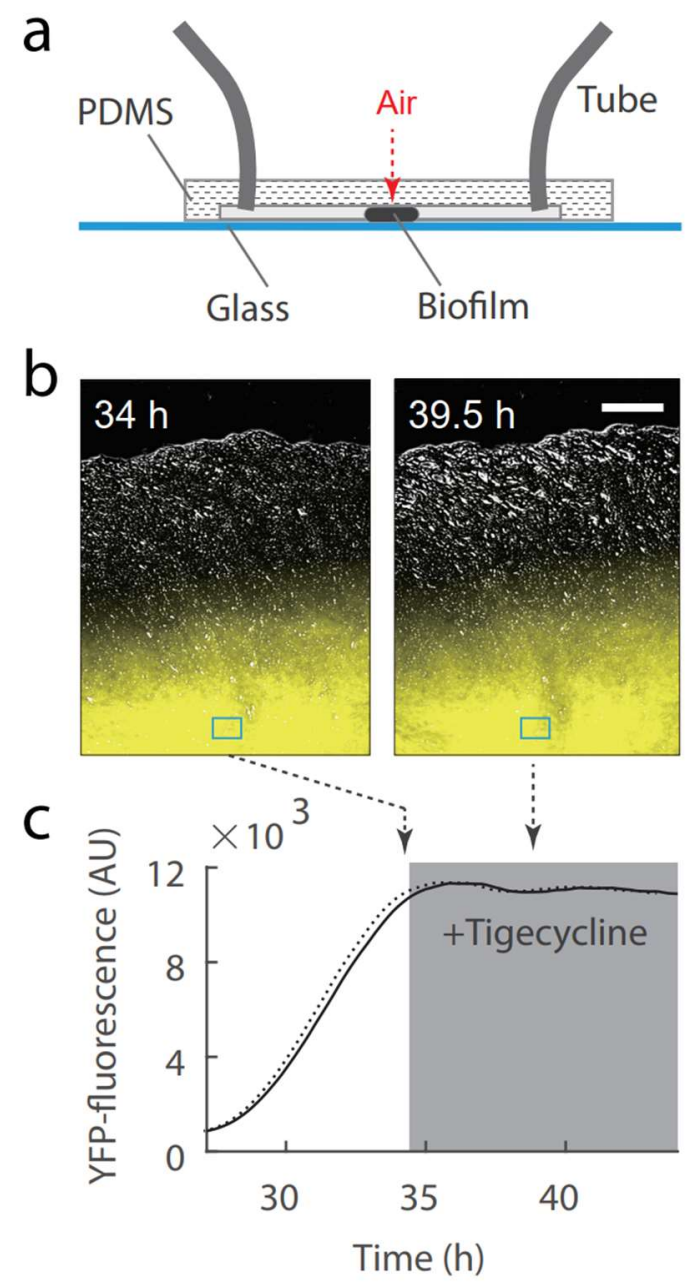

527 Supplementary Fig. 2: Biofilm without oxygen limitation. a, Side view of the microfluidic chip;

528 The red arrow indicates how oxygen from the ambient air could diffuse through PDMS to

529 biofilm interior. b, Snapshots of an E. coli biofilm; Composite of phase contrast and YFP (PptsG-

$530 y f p$ fluorescence, shown in yellow) channels; Scale bar, $100 \mu \mathrm{m}$. c, YFP fluorescence intensity

531 at biofilm interior (the region marked by rectangle in $\mathbf{b}$; The solid line shows the measured

532 fluorescence value; The dashed line shows the estimated level of YFP protein inferred from

533 the solid line, based on the fact that the maturation time of EYFP is 9 mins; The shaded region

534 represents the duration of tigecycline $(2 \mu \mathrm{g} / \mathrm{ml})$ treatments; The dashed arrows indicate the

535 time when the snapshots in $\mathbf{b}$ were taken. 
a
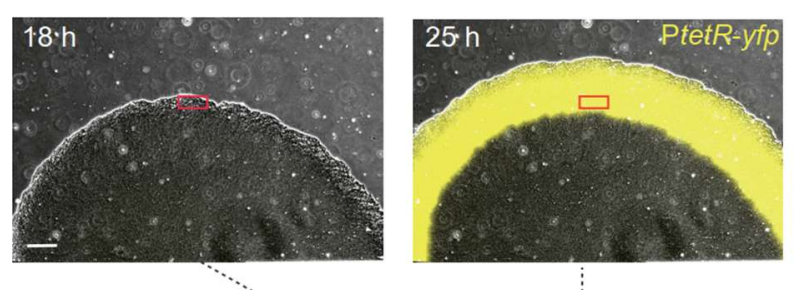

b

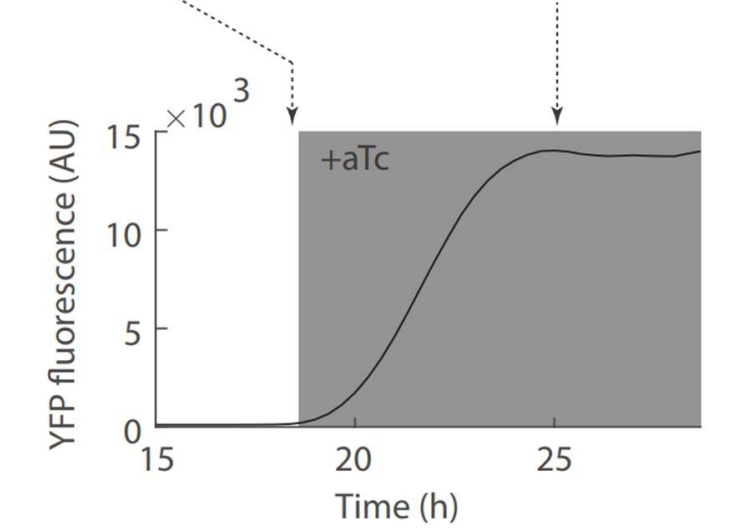

538 Supplementary Fig. 3: Maturation of YFP protein at biofilm periphery. a, Snapshots of an $E$.

539 coli biofilm; Composite of phase contrast and YFP (PtetR-yfp fluorescence, shown in yellow)

540 channels; Scale bar, $100 \mu \mathrm{m}$. b, YFP fluorescence at biofilm periphery (the region marked by

541 rectangle in a); The gray shading represents the duration of aTc (anhydrotetracycline, inducer

542 of the tetR promoter, $100 \mathrm{ng} / \mathrm{ml}$ ) treatment; The dashed arrows indicate the time when the

543 snapshots in a were taken.

544 
a

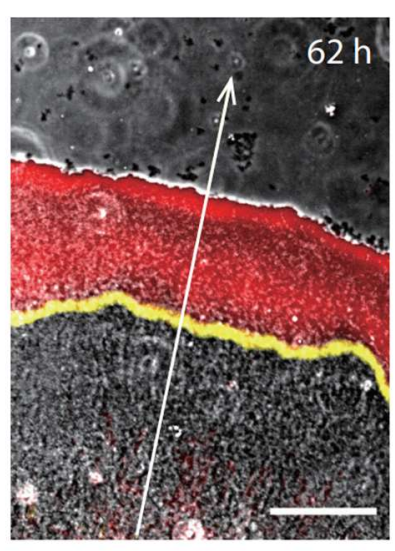

b

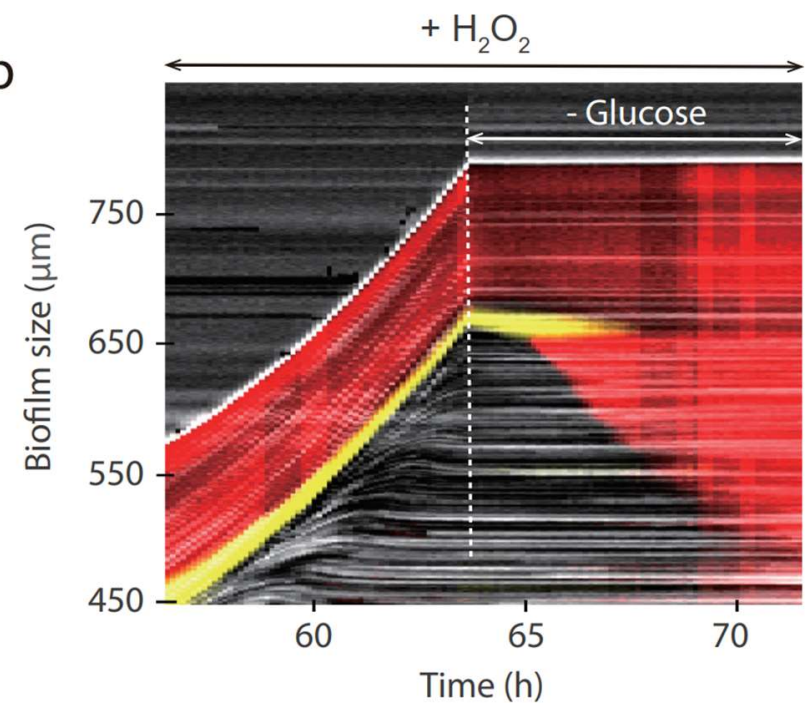

545

546

547 Composite of phase contrast, YFP (DCFH-DA fluorescence, shown in red), and RFP (PkatG-mrfp

549 to glucose removal from the flowing medium; Measurements were taken along the line as 550 indicated by the arrow in a.

551

552
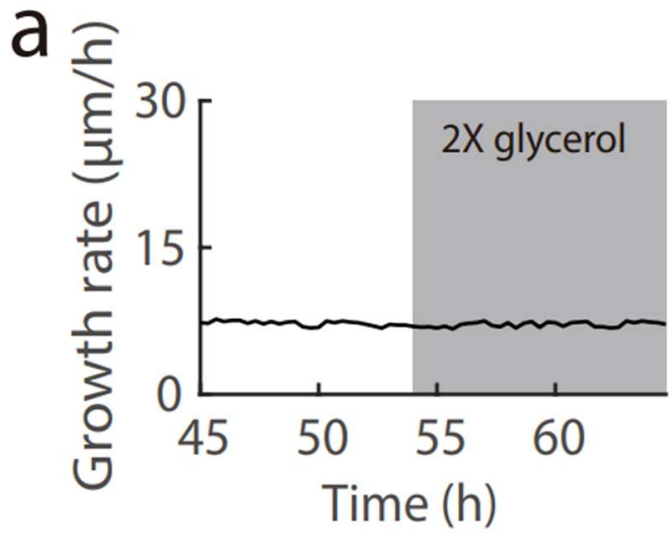

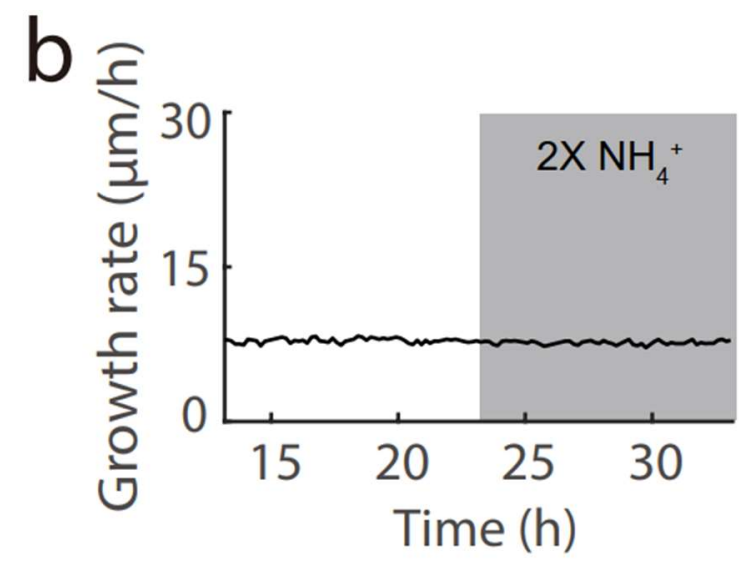

553 Supplementary Fig. 5: Growth of $E$. coli biofilm in modified M63B1. Glycerol (44 mM) instead

554 of glucose was used as the carbon source. a, Biofilm growth rate before and after switching 
a
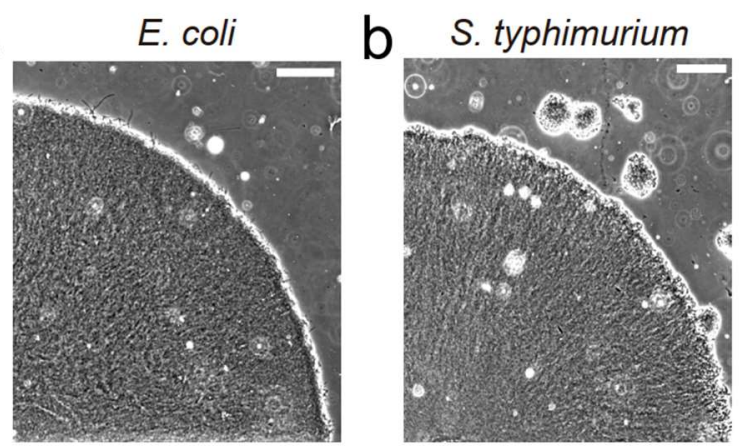

e
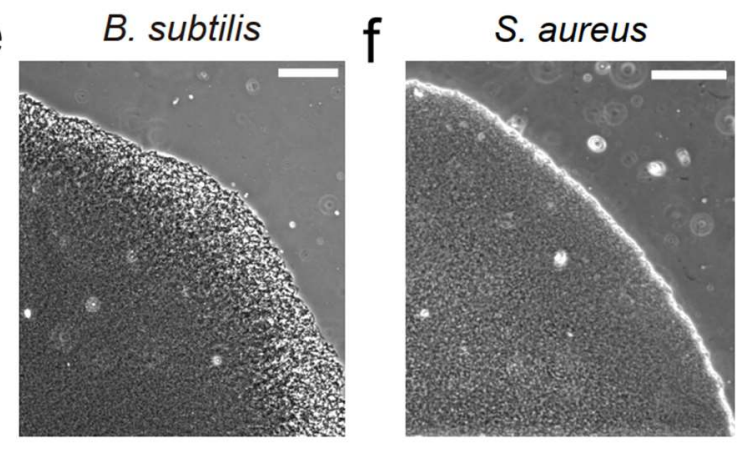
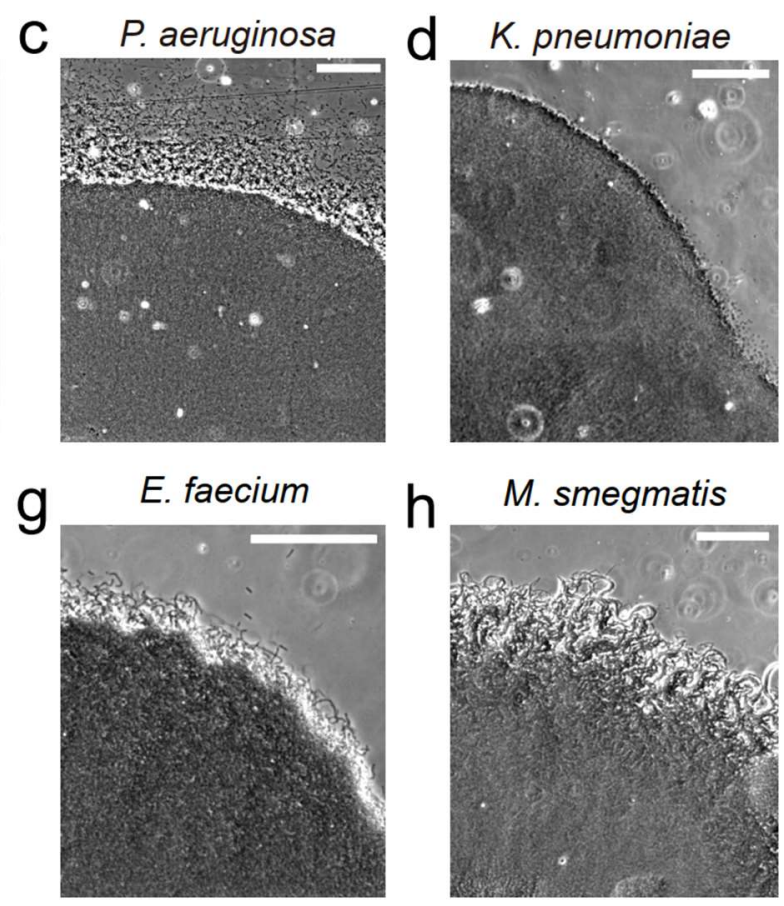

557

Supplementary Fig. 6: Phase contrast images of biofilms formed by commonly studied

559

560

561

562

563

564

565

566

culture supernatant.

\section{Ex Em}

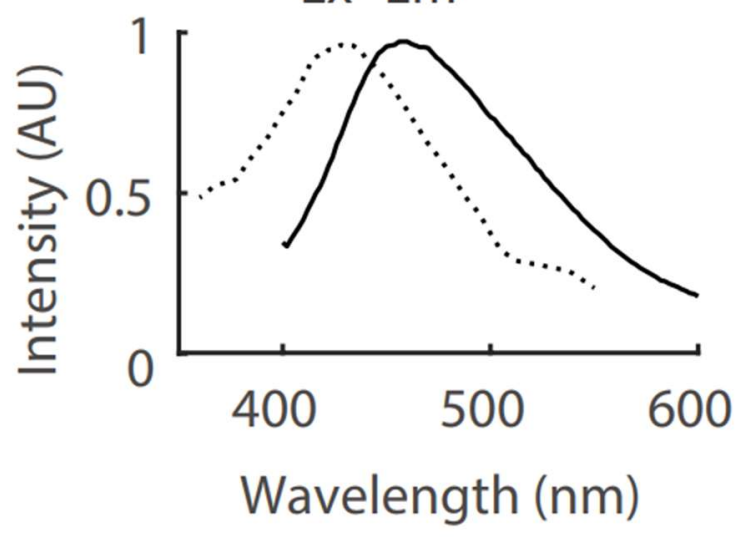



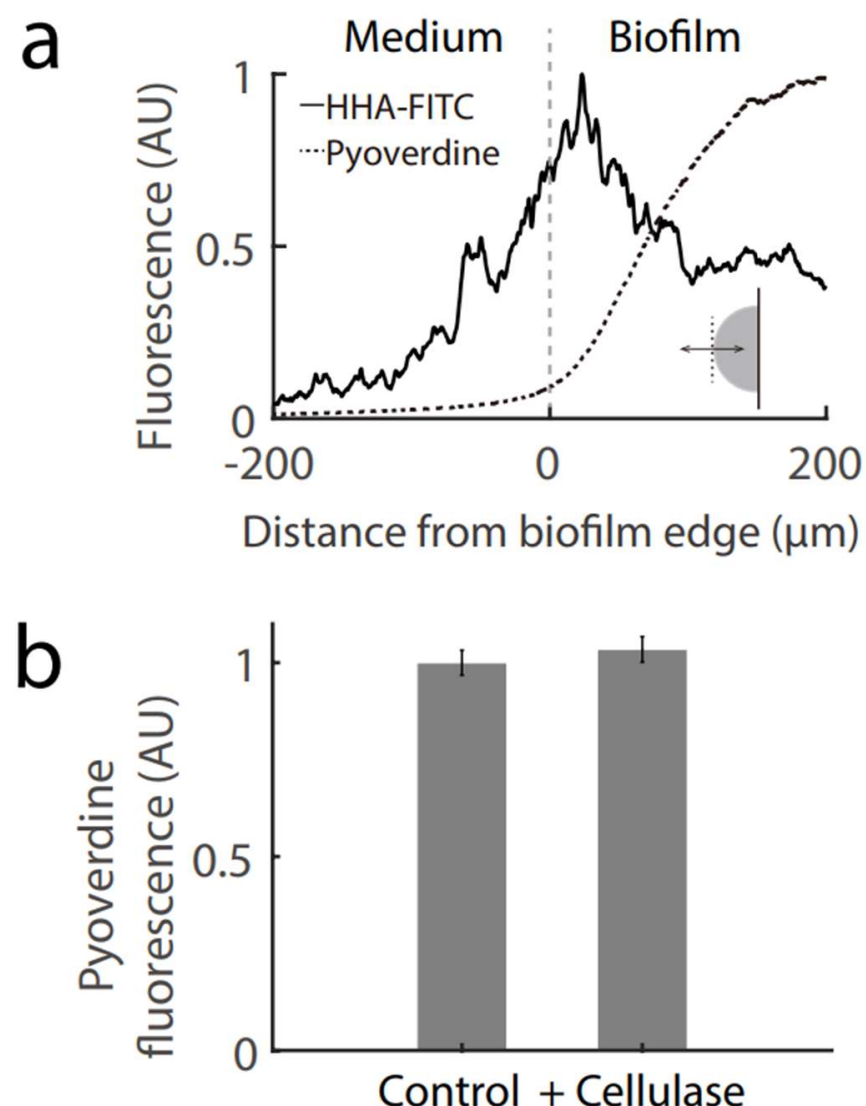

569 Supplementary Fig. 8: Characterization of PsI and pyoverdine. a, Spatial profile of HHA-FITC

570 and pyoverdine fluorescence over a $P$. aeruginosa biofilm; The double arrow in the inset

571 illustrates the line along which the profile was measured. $\mathbf{b}$, Effect of cellulase addition (84

$572 \mathrm{U} / \mathrm{ml}$ ) on pyoverdine fluorescence in stationary phase $P$. aeruginosa culture. The error bars

573 represent standard deviation, $\mathrm{n}=3$ biological replicates. 


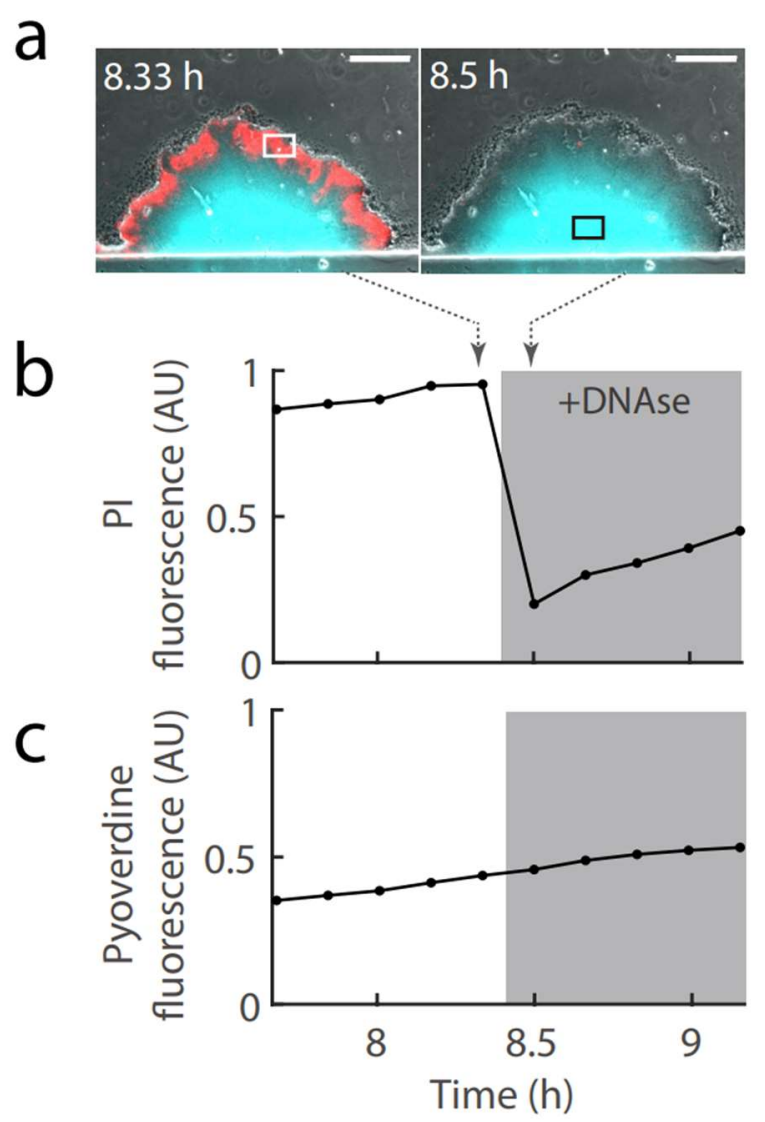

576 Supplementary Fig. 9: Treatment of $\boldsymbol{P}$. aeruginosa biofilm with DNase. a, Snapshots of the

577 biofilm; Composite of phase contrast, CFP (pyoverdine fluorescence, shown in cyan), and RFP

578 (PI - propidium lodide fluorescence, shown in red) channels; Scale bar, $100 \mu \mathrm{m}$. b, PI

579 fluorescence at biofilm periphery as indicated by the white rectangle in a; The gray shading

580 indicates duration of DNase treatment $(750 \mathrm{U} / \mathrm{ml})$; The dashed arrows indicate the time when

581 the snapshots in a were taken. c, Pyoverdine fluorescence at biofilm interior as indicated by

582 the black rectangle in a. 
a

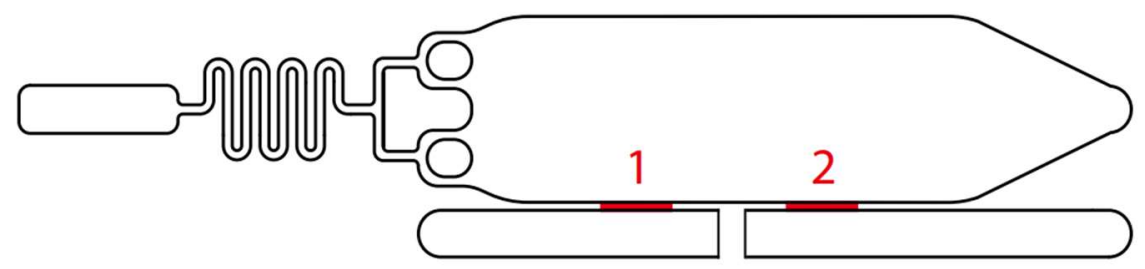

584

b

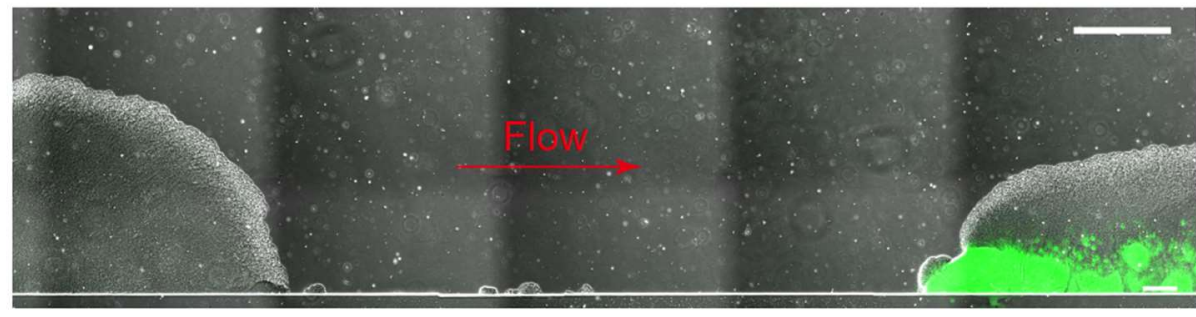

585

Supplementary Fig. 10: Co-culture of biofilms formed by different bacterial species. a,

Schematic diagram of the microfluidic chip; The bacteria seeding zones are marked in red. $\mathbf{b}$,

587

588

589

590

591

592

593

594

595

Snapshot of two biofilms formed by E. coli and P. aeruginosa; Composite of phase contrast and GFP (indicates $P$. aeruginosa, shown in green) channels; $E$. coli and $P$. aeruginosa were loaded to the seeding zones 1 and 2 respectively, and cultured with the M63B1 medium; Scale bar, $300 \mu \mathrm{m}$.

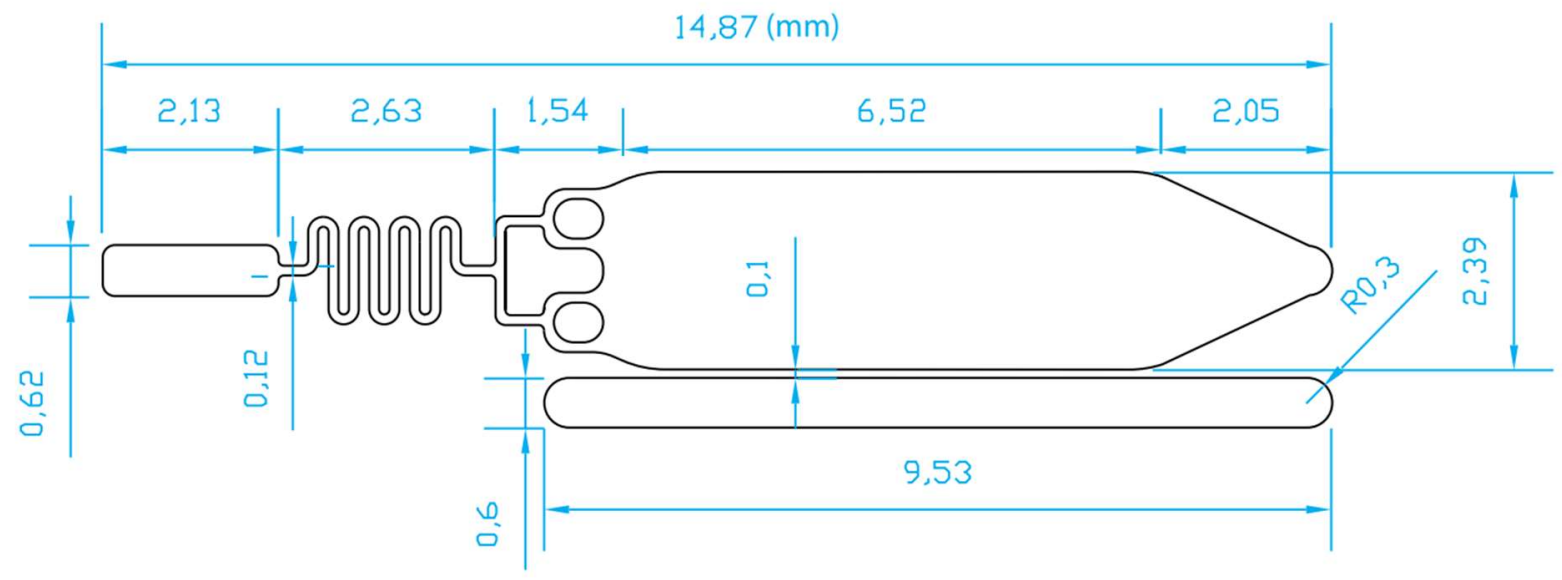

Supplementary Fig. 11: Dimensions of the microfluidic chip introduced in Fig. 1a. 
Supplementary Video 1: Time-lapse video of an E. coli biofilm.

599

600 Supplementary Video 2: Time-lapse video of a $\boldsymbol{P}$. aeruginosa biofilm. Composite of phase

601 contrast and CFP (shown in cyan) channels;

602

603 Supplementary Video 3: Time-lapse video of a $\boldsymbol{P}$. aeruginosa biofilm. Composite of phase contrast, CFP (pyoverdine fluorescence, shown in cyan), and GFP (HHA-FITC fluorescence, shown in red) channels; Switched to medium containing cellulose $(84 \mathrm{U} / \mathrm{ml})$ after $7 \mathrm{~h} 20 \mathrm{~m}$.

606

Supplementary Table 1: Bacterial Strains used in this study

\begin{tabular}{|l|l|}
\hline Strain & Description \\
\hline E. coli & BW25113 \\
\hline E. coli with plasmid, pDL30 PptsG-yfp & PptsG was activated by glucose limited \\
\hline E. coli with plasmid, p15A PkatG-rfp & PkatG was activated by $\mathrm{H}_{2} \mathrm{O}_{2}$ \\
\hline E. coli with plasmid, pSB1C3 PtetR-yfp & PtetR was induced by aTc \\
\hline Pseudomonas aeruginosa & PAO1 \\
\hline Pseudomonas aeruginosa $\Delta p a a P$ & Less prone to dispersion in our experiments \\
\hline Pseudomonas aeruginosa $\Delta p a a P \Delta p v d A$ & Deficient in pyoverdine synthesis \\
\hline Salmonella typhimurium & ATCC 14028 \\
\hline Bacillus subtilis & NCIB 3610 \\
\hline Klebsiella pneumoniae & ATCC BAA-1144 \\
\hline Staphylococcus aureus & RN4220 \\
\hline Enterococcus faecium & ATCC 35667 \\
\hline Mycobacterium smegmatis & mc 2 -155 \\
\hline
\end{tabular}

